\title{
The significant impact of a set of topologies on wireless sensor networks
}

\author{
Lutful Karim $^{1 *}$, Tarek El Salti ${ }^{1}$, Nidal Nasser ${ }^{2}$ and Qusay H Mahmoud ${ }^{1}$
}

\begin{abstract}
Routing and topology control for Wireless Sensor Networks (WSNs) is significantly important to achieve energy efficiency in resource-constrained WSNs, and high-speed packet delivery. In this article, we introduce a framework for WSN that combines three design approaches: (1) clustering, (2) routing, and (3) topology control. In this framework, we implement an energy-efficient zone-based topology and routing protocol. The framework features a new set of graphs referred to as the Mini Gabriel (MG) graphs. The simulation results demonstrate that the framework with the MG graphs and without these graphs are generally $28 \%$ better than the framework with an existing geometric graph. This is in terms of the total network energy consumptions. In addition, the proposed framework is $10,25,26$, and $46 \%$ better than the proposed work with an existing geometric graph in terms of the end-to-end data transmission delay, the transmission energy consumptions, the number of hops in established paths and the routing delay, respectively. Moreover, the MG demonstrates that it achieves the connectivity property, which is critical for WSNs.
\end{abstract}

Keywords: wireless sensor network, topology control, unit disk graph, Gabriel graph, routing protocol

\section{Introduction}

Wireless Sensor Networks (WSNs) have many interesting applications such as disaster detection and monitoring. A WSN is a type of an adhoc network that consists of a collection of sensor nodes. Each of these nodes has several capabilities (e.g., sensing temperature) that allow it to gather the information about the surrounding environment. The nodes send all captured data to a Base Station (BS) for processing and further analysis.

Several challenging design factors exist for WSN. In this article, we focus on routing and topology control challenges. Many of the existing routing protocols [1-3] use energy-inefficient tree structure and dynamic flooding. These protocols use the number of hops as the length of data transmission path. However, the actual length of the shortest hop path can be longer than that of a larger hop path. Other existing protocols $[4,5]$ enable the senor nodes to exchange a great number of control messages. Hence, the WSN's lifetime is dramatically shortened. Moreover, there are routing protocols [6] that use the global topological information for

\footnotetext{
* Correspondence: Ikarim@uoguelph.ca

'School of Computer Science, University of Guelph, Guelph, ON, Canada Full list of author information is available at the end of the article
}

message forwarding where sensor nodes have limited resources such as memory size. Combining all these drawbacks leads to the following research question: how to construct a routing protocol that has the following interesting behaviors: (1) being efficient in terms of the power consumption, and (2) using the local neighborhood's information for packet forwarding.

Related to this problem is the topology control challenge for WSN where several algorithms are used [7-9]. However, the constructed topologies remove too many edges (i.e., wireless links). Furthermore, some of these graphs (i.e., the Gabriel Graph (GG) $[7,10]$ and the Relative Neighborhood Graph (RNG) [9]) are planar graphs. As a consequence, many shortest paths are discarded. Thus, the packet delivery speed is dramatically reduced [11]. In addition, the power consumption for the source-destination paths is significantly increased. This introduces another challenge: how to maintain as much of the shortest paths as possible when new topologies are constructed. To the best the authors' knowledge, there is no existing work that addresses all these challenges in one framework. In addition, this integration allows us to investigate how several approaches interact in terms of several metrics over existing approaches. 
In this article, we introduce a framework for WSN that combines three design approaches: (1) clustering, (2) routing, and (3) topology control. We call the framework Cluster-based Routing and Topology Control Approach (CRTCA). In this framework, we implement an energy-efficient zone-based topology and routing protocol. Afterwards, we present a new set of graphs referred to as the Mini Gabriel (MG) graphs. The simulation results show that the framework based on the new set of graphs, i.e., MG outperforms the Gabriel Graph GG $[7,10]$. This is in terms of the total network energy consumptions, transmission energy consumptions, routing delay, and end-to-end data transmission delay. In addition, the proposed framework generally demonstrates the best performance in terms of the most performance metrics except data transmission delay where both CRTCA and CRTCA-MG at lower values are considered to be the same. Moreover, the MG demonstrates that it achieves the connectivity property. Achieving this property is critical for WSNs.

\subsection{Assumptions and terminologies}

The reasons for choosing GG in our comparisons are as follows. First, MG set's elimination areas are derived from GG's elimination area. Second, GG has been generally used as underlying topologies for WSNs [12]. Third, GG reduces the degree of nodes but does not remove too many edges compared to other existing geometric graphs $[8,9]$. Therefore, GG preserves shortest and least power paths compared to those existing graphs.

In this article, all the sensor nodes in the network are assumed to run a sleep scheduling algorithm [13] to manage their residual energy. In addition, those nodes have the same sensing range $\left(R_{\mathrm{s}}\right)$ and communication range $\left(R_{\mathrm{c}}\right)$ (i.e., the network is homogenous). Furthermore, each sensor node has its 2D geometric position and its neighbors' positions [14]. From these positions, the 2D Euclidean distance between any pair of sensor nodes, say $u$ and $v$, is denoted by $|u v|$. Any pair of sensor nodes communicates if the distance between this pair is less than the communication range (i.e., Unit Disk Graph-UDG). This graph, denoted by $G$, is represented by a set of vertices (or sensor nodes) $|V|$ and a set of edges (or wireless links) $|E|$. In other words, any pair of sensor nodes communicates if their sensing circles intersect by at least one point. This is based on the double range property [15], i.e., $R_{\mathrm{c}}=w . R_{\mathrm{s}}$, where $w \geq 2$. The term $w$ is a constant parameter. The WSN is divided into clusters (or zones). Each zone has at least one Cluster Head $(\mathrm{CH})$ and one active sensor node. All these nodes are uniformly distributed into each zone. Moreover, each zone is specified by the number of hops away from the BS. For instance, sensor nodes whose hop count is less than or equal to three reside in a specific zone. Each zone is divided by the BS into squares where there is at least one sensor node into each square. Notice that the $R_{\mathrm{s}}$ of a sensor node located in a square is $2 \sqrt{2}$ times the length of that square side. All these assumptions are necessary for the proposed work where this is the first time that clustering, routing, and topology control are all investigated as one main framework. The proposed work does not consider mobile sensor nodes just fixed nodes.

The remainder of this article is organized as follows. Section 2 discusses work related to topologies, routing protocols, and geometric graphs. Section 3 proposes the CRTCA framework that uses Gabriel and MG graphs. Section 4 presents the evaluation results and analysis of CRTCA, and finally, Section 5 concludes the article and presents ideas for future work.

\section{Literature review}

We study the topologies for WSN, which are used in existing monitoring applications. Kait et al. [5] propose a WSN-based paddy growth monitoring system. Sensor nodes gather and send field data, such as temperature, periodically to the BS. The procedure is done by using multi-hop routing which is not considered energy efficient. This is because sensor nodes transmit data through the nearest neighbor which might end up with the longest path. Moreover, this routing protocol does not consider the energy level of the sensor nodes to generate transmission path. Another interesting study by Yoo et al. [3] proposes a precision and intelligence agricultural system referred to as the Automated Agriculture System. The goal of this system is to monitor and control the growing process of melon and cabbage in a greenhouse. In the system, sensor nodes are organized in a parent-child tree structure. The nodes join the network by broadcasting a parent search packet. Furthermore, the nodes transmit data to the BS using three gateway nodes. However, the tree structure has a single point of failure. Yang et al. [16] developed an intensive WSN-based irrigation monitoring system. Sensor nodes are placed by this system in widely separated clusters. Thus, sensor nodes consume much energy for transmitting data to remote nodes in other clusters.

Chitiet al. [1] propose next generation firm for Agrofood productions. This system uses Ambient Intelligence and WSN. Moreover, the proposed system provides feedback and adaptability to increase productions in Agro-food. However, the deployed WSN uses a dynamic flooding inefficient-energy routing protocol. This is due to the fact that a large number of messages are broadcasted. Village eScience for Life [4] is a WSN-based agriculture project. It is implemented in developing 
regions in Africa and uses dynamic zone-based topology. This project initially deploy sensor nodes into zones in such a way that each sensor node remains within the transmission range of the nodes of at least two zones and each node belonging to a zone elects nodes in neighboring zones to which it can connect with a minimum transceiver power. Hence, several graphs are generated and the graph requiring minimum transmission power is selected for routing. However, this routing protocol does not guarantee to eliminate sensing holes. COMMONSense Net (CNS) [2] is another WSN-based agriculture monitoring project developed for semiarid regions in developing countries. The routing protocol of CNS uses tree structure which is not reliable since a link failure or sensor node failure can make other nodes unreachable to BS.

Unlike the earlier works that focus mainly on the WSN-based monitoring applications, recent research [8] has significantly considered studying the actual structure of WSN through graph theory. In particular, geometric graphs are used in WSNs [17] to model the relationship between a sensor node and its neighboring sensor nodes. The GG $[7,10]$ uses a forbidden area to define the edges created between a set of sensor nodes. The constructed edges are undirected. In addition, the GG is a sub-graph of the UDG, where the UDG is connected. The algorithm for constructing the GG is as follows (see
Figure 1). An edge binding two vertices, say $v_{1}$ (i.e., running the algorithm) and $v_{2}$ is in GG if the following criterion holds: the minimal diameter circle that circumscribes $v_{1}$ and $v_{2}$ whose diameter is the line segment that has both $v_{1}$ and $v_{2}$ as its endpoints has no other vertex of $V$. The sensor node $v_{1}$ follows these steps with the other neighbors. Moreover, the algorithm is executed on every sensor node in the network.

Similar to the GG mentioned above, the RNG [9] also uses a forbidden area to define the edges constructed between a set of sensor nodes, where the edges are considered undirected (see Figure 2). This graph can be considered as a subgraph of GG and UDG graphs, where all these graphs are connected. The algorithm for constructing the RNG graph is as: an edge binding two vertices, $u$ and $v \in V$, is in RNG if the intersection of the two circles centered on two vertices with radii $|u v|$ does not have any other vertex $w \in V$. From Figure 2, the edge is constructed since node $w$ is not in the intersection of the two circles centred on two vertices with radii $|u v|$.

Another interesting graph is the Half Space Proximal (HSP) graph proposed by Chavez et al. [8]. It constructs an edge based on a forbidden area with the nearest neighboring node. There are two versions of this graph: either directed or undirected graphs. The algorithm for constructing this graph is as follows (see Figure 3). First,

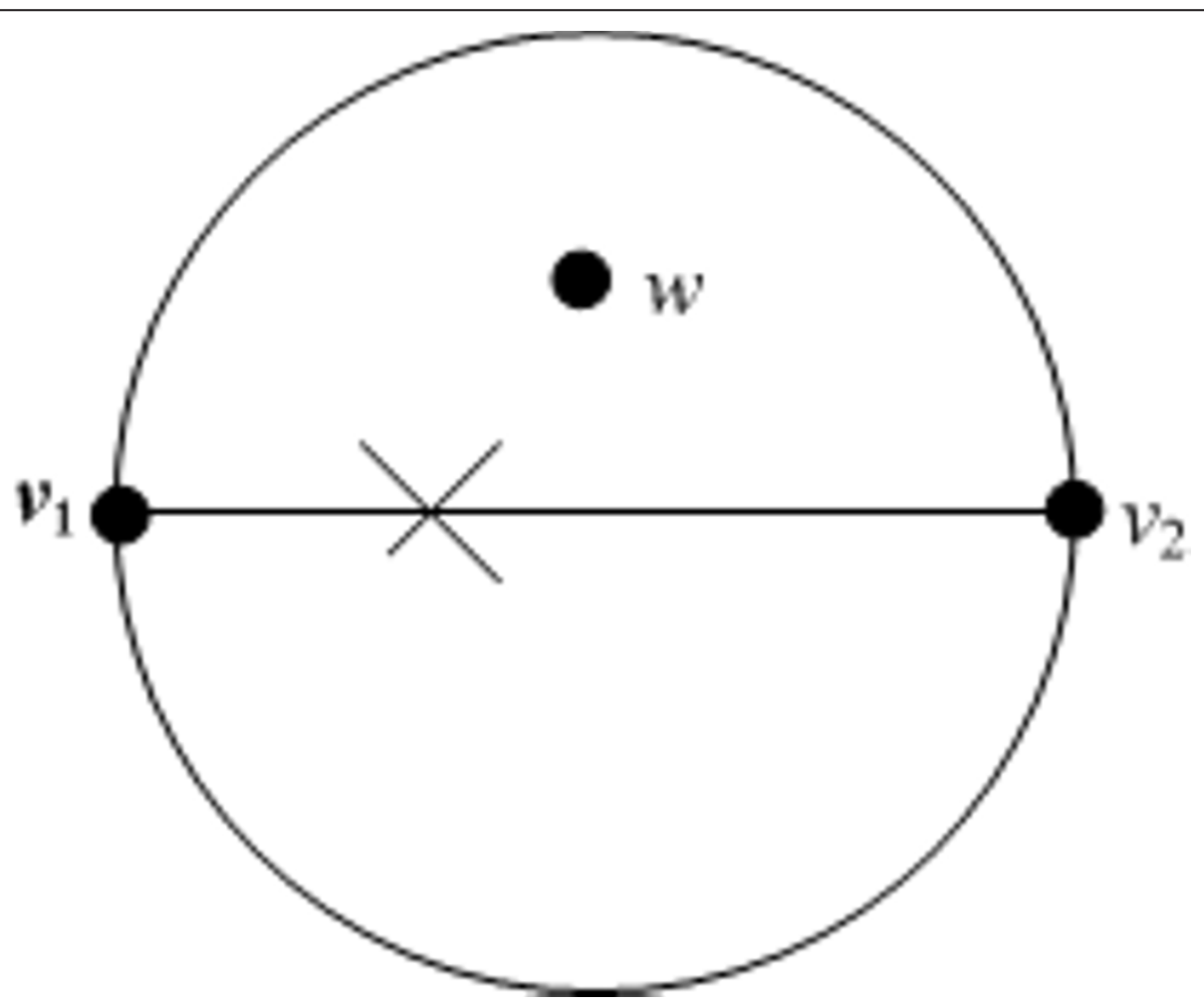

Figure 1 An edge $\left[v_{1}, v_{2}\right]$ is not in GG. 


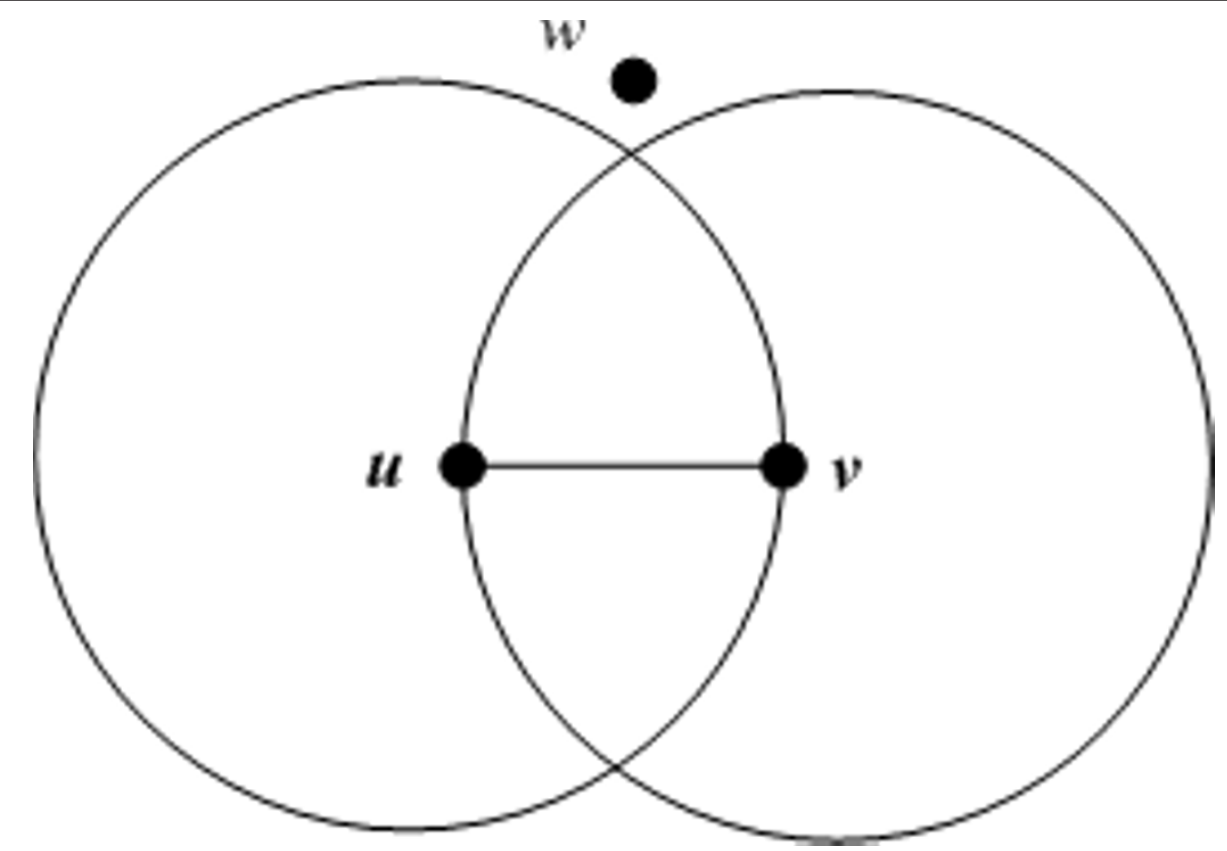

Figure 2 The edge $[u, v]$ is in RNG.

a directed D-HSP $(G)$ is defined, where $G$ is defined to be the UDG and it is connected. At each node $u$ in $G$, the following iterative procedure is performed until all the neighbors of $u$ are either discarded or are connected with an edge. A directed edge $[u, v]$ is formed with the nearest neighbor $v$. An open half plane is defined by a line perpendicular to $[u, v]$, intersecting $[u, v]$ at its midway point, and containing $v$. All the sensor nodes in this half plane are then discarded. The procedure then continues with the next nearest non-discarded neighbor and so on until all the sensor nodes have been discarded. The selected directed edges determine the D-HSP $(G)$.

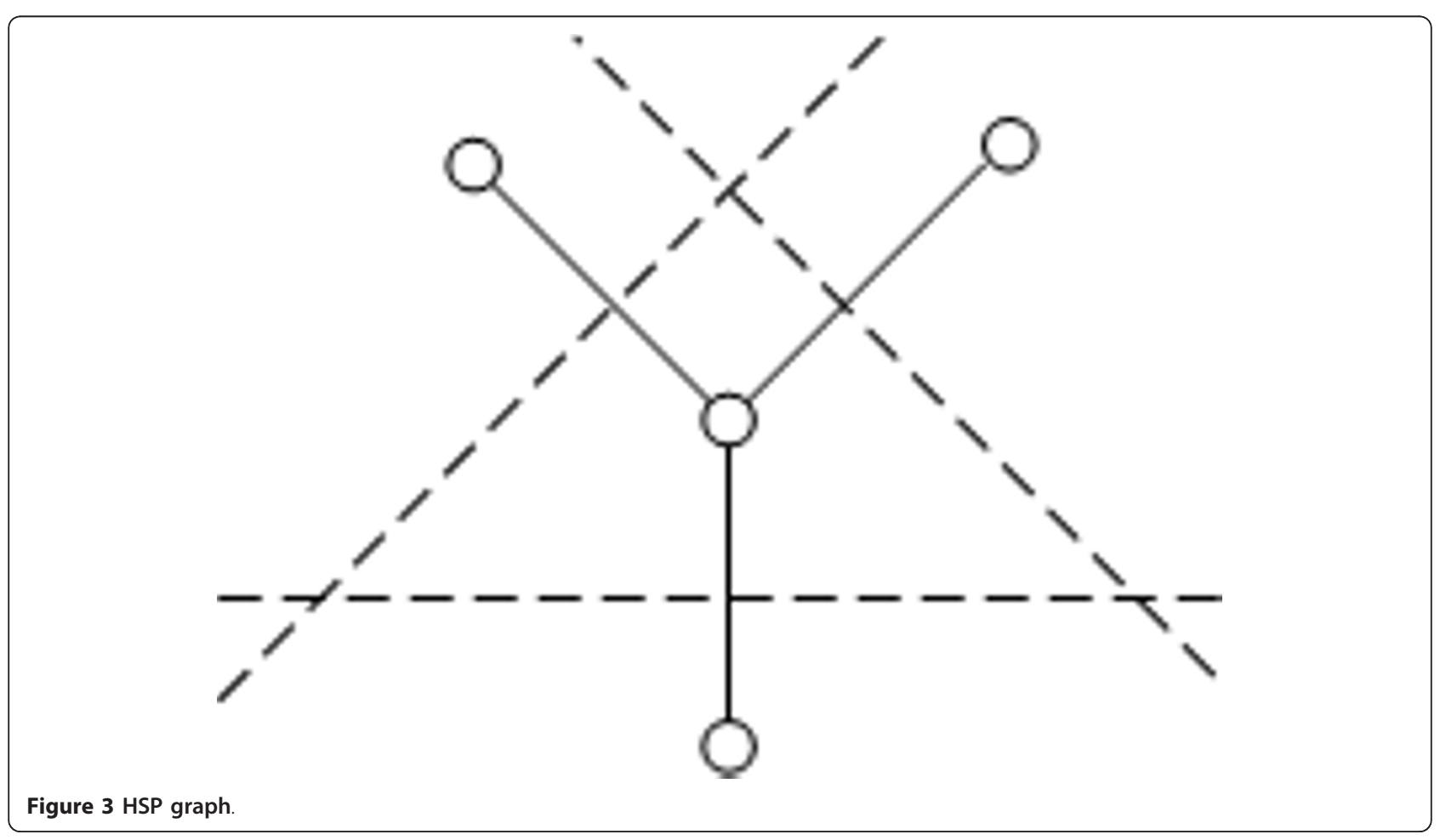


The undirected HSP $(G)$ is obtained by ignoring the direction of the edges.

In general, all the mentioned graphs reduce the degree of the sensor nodes to shorten the routing delay on each node. As a consequence, many shortest paths in terms of the Euclidean distances are discarded between any pair of sensor nodes. Moreover, some least power paths between sensor nodes are unfortunately lost. Furthermore, Shu et al. [11] mention that although GG and RNG are planar graphs, the delay for the multimedia WSNs based on these graphs is dramatically increased. As a summary, Table 1 demonstrates the differences between all the mentioned graphs in terms of different behaviors and definitions.

The reasons that GG eliminates few edges compared to RNG and HSP are as follows. First, GG has a bounded elimination area (i.e., circle) compared to the HSP (i.e., open half plane). Second, GG's circular forbidden area exists in the RNG's elimination area (i.e., in the forbidden area constructed from the intersected of two circular areas).

\section{An integration of clustering, routing, and topology control approaches}

In this section, we introduce an integrated framework referred to as the CRTCA for WSNs. We incrementally integrate the proposed clustering, routing, and topology control approaches to construct the framework. We begin with the zone creation and active nodes selection that is followed by establishing paths among active nodes to the BS. Each of the integrated components is presented in the following sections.

\subsection{Zone creation and active node selection}

Once sensors are localized, the network area is divided into a number of zones. The BS assigns the sensor nodes to different zones based on their geometric positions.

For instance, if the network area is very small (e.g., $100 \mathrm{~m} \times 100 \mathrm{~m}$ ) and divided into four zones, then sensor nodes are distributed as follows. Nodes with positions between $(0,0)$ and $(50,50)$ reside in Zone 1. Whereas sensor nodes with positions between $(50,0)$ and $(100,50)$ reside in Zone 2 . Figure 4 illustrates the zones construction where the BS is located outside of those zones.

Table 1 Differences between GG, RNG, and HSP graphs

\begin{tabular}{lll}
\hline Graph type & Elimination area & Sensor nodes eliminations \\
\hline GG $[10,16]$ & $B$ & Less than HSP and RNG \\
RNG $[8]$ & $B$ & More than GG \\
HSP [6] & $U$ & More than GG and RNG \\
\hline
\end{tabular}

$\mathrm{B}$, bounded; $\mathrm{U}$, unbounded.
Afterwards, a minimum number of active sensor nodes are chosen by the BS and it works as follows. Each zone is divided into squares where each square has at least one active sensor node. However, even if this assumption does not hold, the region of that square is sensed by an active node of the neighboring squares. This is because an active sensor node in a square has the sensing coverage of all the neighboring squares (Figure 5). Therefore, a very high probability of not having a sensing hole in the network is achieved. As a result, fault tolerance is accomplished. For instance, whenever the active sensor node of a square $g_{1}$ fails, the network operation (i.e., sending and receiving messages) proceeds. This is done without activating another sensor node for that square or re-establishing the network topology. This is because the active node of the neighboring square of $g_{1}$ fully covers $g_{1}$.

Using this topological structure, there is a high probability that no sensing hole exists in the network if (see Figure 5). The terms $h$ and $R_{\mathrm{s}}$ is the side of a square and the sensing range, respectively. Let us consider the following. The active sensor node $a_{1}$ of square $g_{1}$ exists at the bottom left corner and the active node $a_{2}$ of square $g_{2}$ exists at the top right corner. If the node $a_{2}$ fails, then the square $g_{2}$ can still be covered by the node $a_{1}$ of square $g_{1}$. This is even if no other neighboring square of $g_{2}$ has active node. This is explained as follows. The farthest point $p_{2}$ of $g_{2}$ is within the sensing range of $a_{1}$. By following Pythagoras formula, the square coverage is possible. Figure 5 also clarifies this relationship between $R_{\mathrm{s}}$ and $h$.

This topological structure also ensures that several active sensor nodes (i.e., at least one sensor node) of a zone reside within the communication range $\left(R_{\mathrm{c}}\right)$ of the active nodes of the neighboring zones. This enables the sensor nodes to transmit the data to the BS through active nodes in their neighboring zones. This is because $R_{\mathrm{c}}>R_{\mathrm{s}}$ and there is a defined relationship between those ranges [1]. In Figure 6, we find that sensor node $a_{2}$ is at the border of two zones $Z_{i}$ and $Z_{i+1}$. Sensor nodes $a_{1}$ and $a_{2}$ are at $R_{\mathrm{s}}$ apart. In addition, sensor nodes $a_{1}$ and $a_{3}$ are at $R_{\mathrm{c}}$ apart. In addition, a large number of nodes in $Z_{i+1}$ reside within the $R_{\mathrm{c}}$ of node $a_{1}$ of Zone $Z_{i}$. Using this topological structure even if a small number of sensor nodes are distributed into the zones, the network is still expected to be covered. The reason is that each sensor node in a square covers all surrounding squares.

The BS chooses a $\mathrm{CH}$ for each zone. The chosen node is responsible for controlling the zone as well as selecting active nodes of a zone. The criterion for choosing the $\mathrm{CH}$ is as follows. Based on our assumption that all sensor nodes initially have the same residual energy, the BS randomly chooses a sensor node. The chosen node 


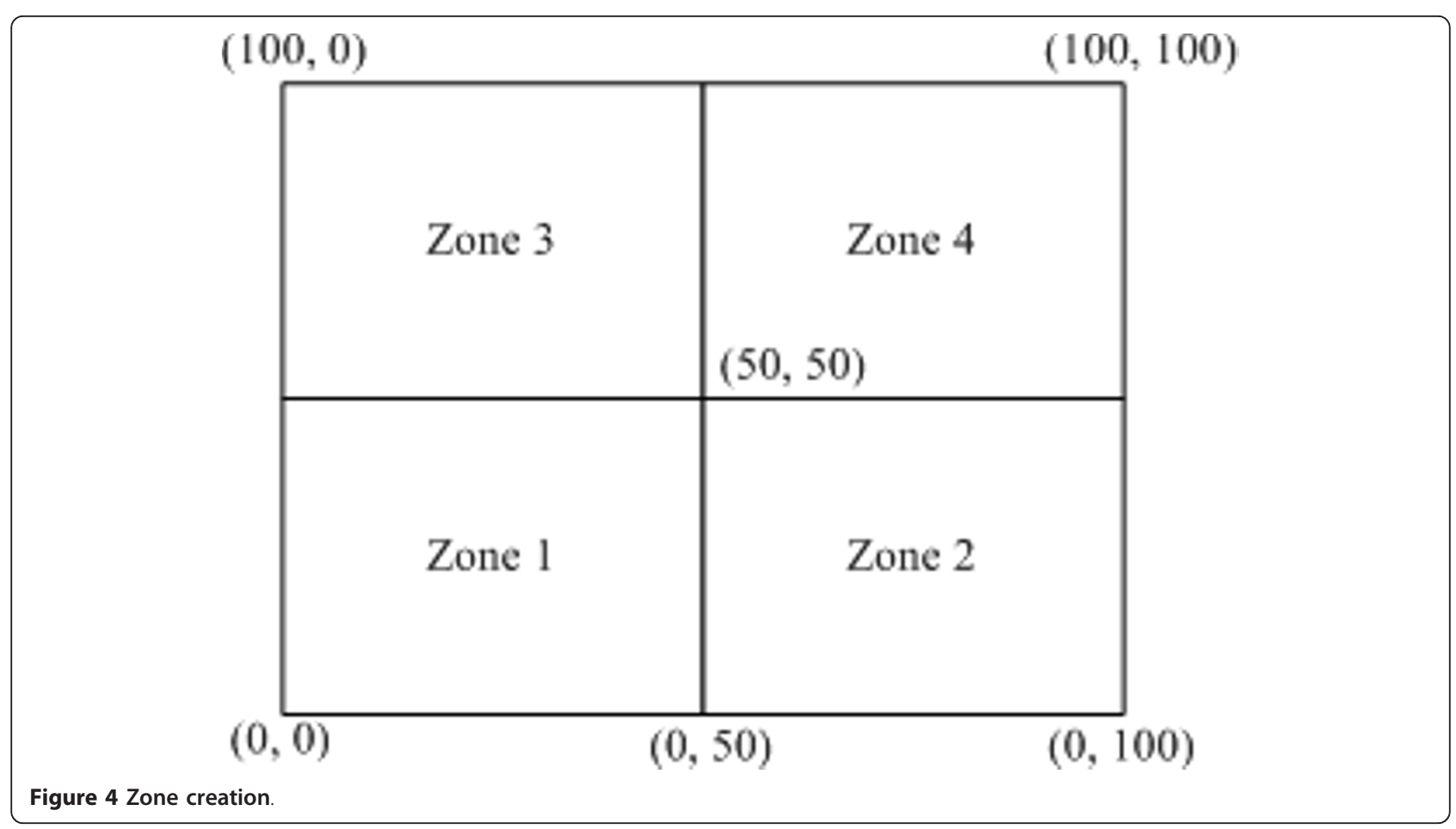

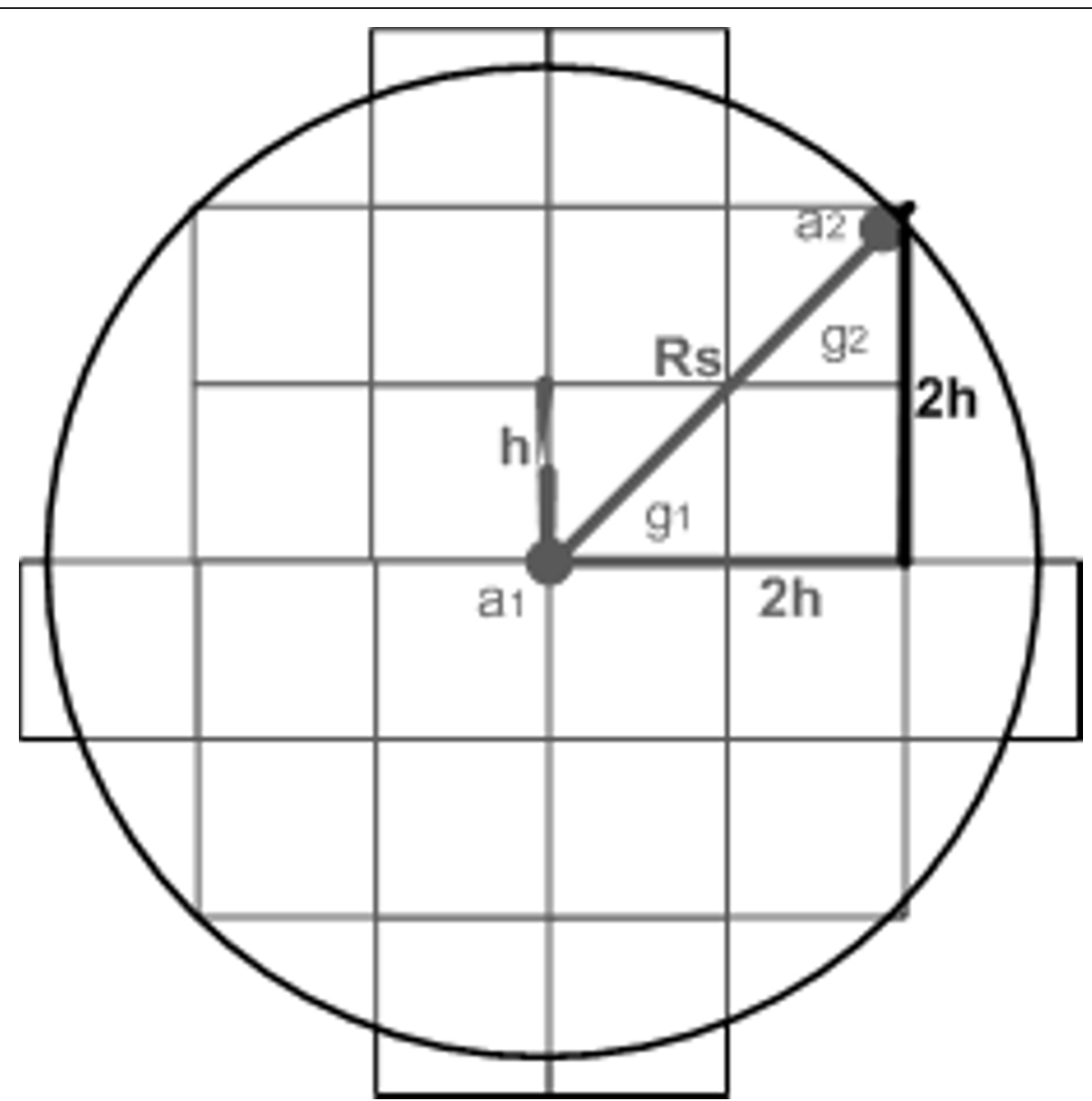

Figure 5 Zone-based topology where the sensing range. $R_{\mathrm{s}}=2 \sqrt{2} \mathrm{~h}$. 


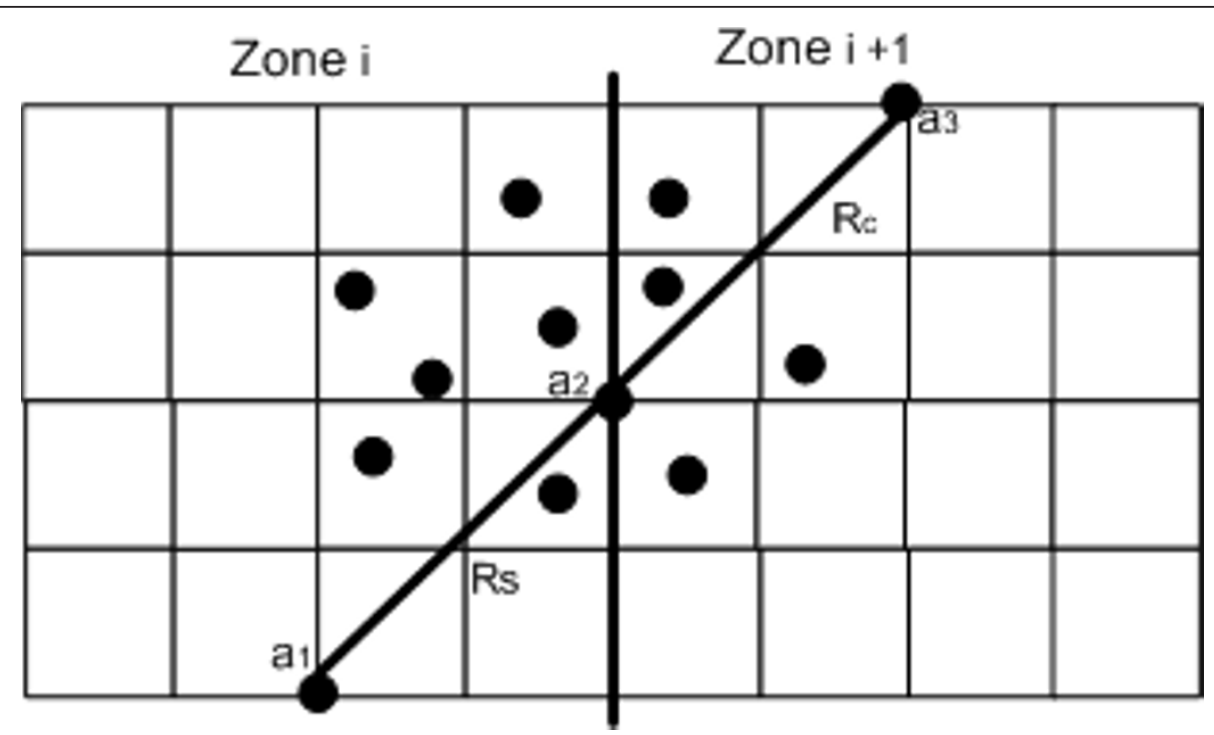

Figure 6 Zone-based topology with several active nodes within communication range of each other when $R_{\mathrm{c}}=n \times R_{\mathrm{s}}, \mathrm{n} \geq 2$.

becomes the $\mathrm{CH}$. If the residual energy of the sensor nodes varies, the sensor node with the highest residual energy is chosen as $\mathrm{CH}$. Then, the $\mathrm{CH}$ chooses active nodes in each zone based on (1) residual energy, (2) distance, (3) number of sleep rounds, and (4) free buffer spaces. If only one sensor node is located in a square, then it is chosen as an active node. Otherwise, having the same residual energy in nodes, say $A$ and $B$ in the same zone, node $A$ is chosen as an active node. This is when node $A$ is closer to the $\mathrm{BS}$ than $B$ or active nodes of neighboring zones. Further ties are broken by comparing the remaining storage space or the number of sleep rounds of a node. Especially, node $A$ is given more priority if it has more remaining storage space compared to node $B$. All other sensor nodes except the chosen active nodes remain in sleep mode. This is done by turning their sensing and transmission radios off. Thus, a number of active nodes are determined in each zone. The determined number of sensor nodes provides a high probability that these nodes provide the sensing coverage of the whole network. Then, each active node generates the shortest path to the $\mathrm{BS}$. Once the $\mathrm{CH}$ and active nodes are chosen, they work for a certain period of time (or rounds). They stop working once their residual energy goes below a threshold value. After the clustering technique is proposed, we proceed with the second component of the proposed framework as follows.

\subsection{Path establishment method}

The path establishment method works as follows. Sensor nodes at level $L_{1}$ calculate their distances to the BS and send this distance information to the sensor nodes at level $L_{2}$. Then, nodes at level $L_{2}$ calculate their distances to the sensor nodes at level $L_{1}$ and then calculate the total distance to the BS. This is done through different active nodes at level $L_{1}$. Thus, active nodes at the level $L_{2}$ find out the shortest path to the BS (see Figure 7).

Similarly, nodes at level $L_{k}$ compute the total distance of the neighboring nodes at the upper level $L_{k-1}$ to the BS. Afterwards, these nodes identify the shortest path to the BS. Based on this information, an active node creates a communication path with the neighboring active nodes. As a result, the total distance to the BS as well as the power consumptions is minimized as compared to existing tree-based routing protocols since power consumptions are proportional to the distance of transmission path.

Moreover, each active sensor node $x$ at the level $L_{i}$ chooses another active sensor node $y$ at the level $L_{i-1}$ with which $x$ has the second lowest distance to the BS. The sensor node $y$ acts as an alternative node or as an intermediate node of an alternative path for each leaf node to the BS. This enables the leaf node to transmit data to the BS in case of any active node fails. Once the paths are established, active nodes sense and route the data to the BS. Figure 7 demonstrates the path establishment method, where the shortest path from a leaf node to the BS is represented by bold arrows. Figure 8 illustrates the alternative path establishment and the transmission of data through alternative node when an active sensor node fails. For instance, if node $K$ at level 1 fails, active sensor node $F$ at level 2 transmits data through node $L$. The alternative path is shown as red arrows. Based on the proposed framework, a new set of graphs for underlying topologies is proposed as follows. 


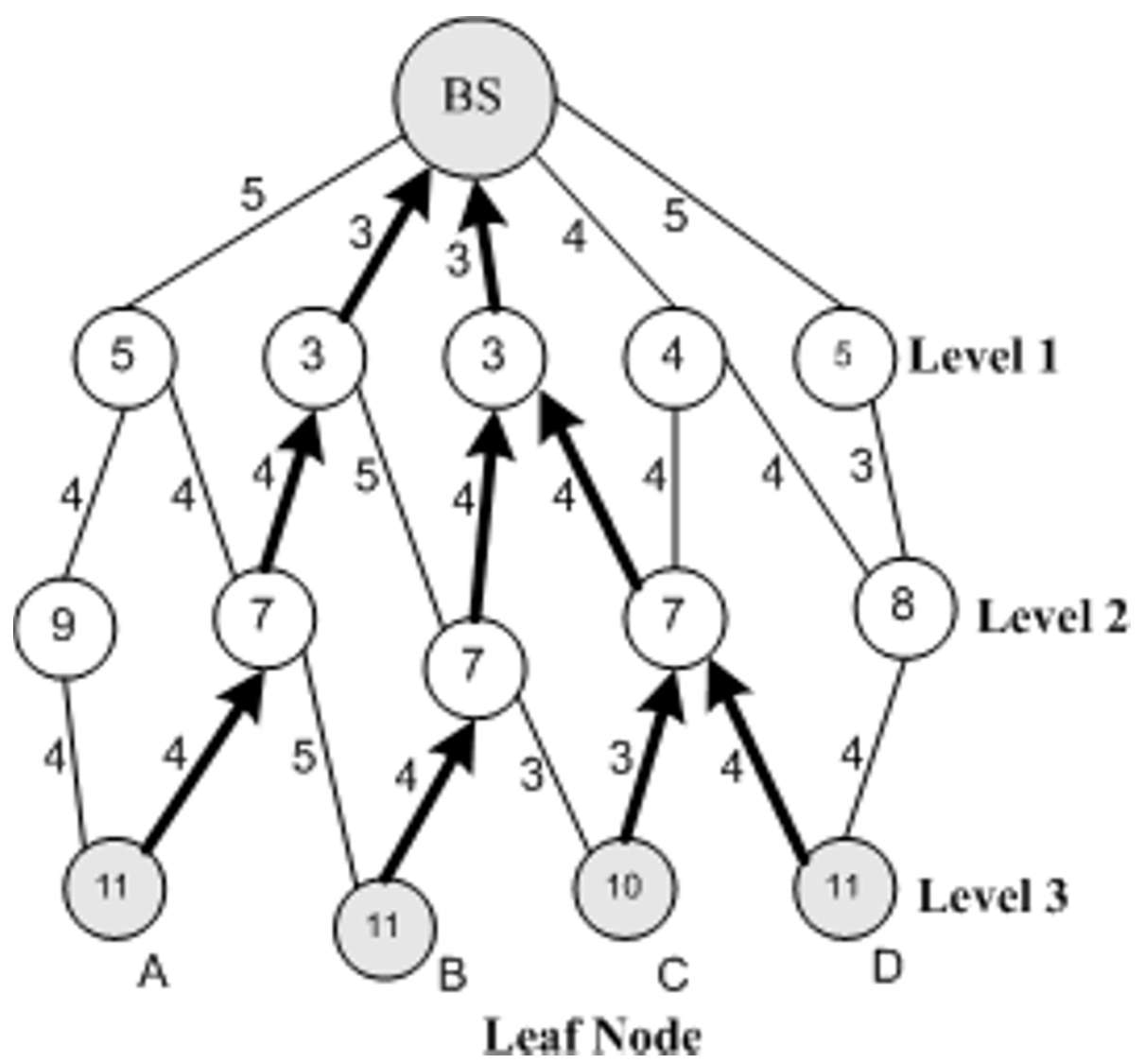

Figure 7 Shortest paths from leaf nodes to BS using path establishment method

\subsection{A set of topology control algorithms}

We introduce a new set of graphs referred to as the MG geometric graphs. These graphs are sub-graphs of the UDGs. Each sensor node, $u$, running these graphs chooses the nearest neighboring node, $v$. Afterwards, a midpoint mid is calculated between the sensor nodes $u$ and $v$. As a result, a circle $c$ is constructed with a center located at mid with a radius $r_{-}$mid equal to $s(|u v| / 2)$. The term $s$ is a constant parameter within the range of $[0,1]$ which shortens or increases the $r$ mid. If $c$ contains a sensor node, say $w$, in its area, then the edge [u, $v]$ is removed. Otherwise, the edge $[u, v]$ is preserved.

The algorithm proceeds on each sensor node until the network originally modeled by the UDG is reconstructed. This construction is based on a given $s$ value. Because the MG only needs the position of the sensor node running this algorithm (i.e., current sensor node) as well as its neighboring nodes, this algorithm is therefore local. If $s$ is equal to1, then a special case of the graph is constructed, a GG $[7,10]$. However, the new set of graphs preserves more edges by using smaller circles and it may have crossing edges (i.e., non-planar graphs if $s \neq 1$ ). This is compared to that circle constructed by the GG. The possibility of maintaining shorter paths is therefore increased in the MG. This is compared to the GG which makes the MG a denser graph. The MG runs in $O\left(l^{*} l\right)$ where $l$ is the degree of sensor nodes. This is because each time a sensor node, say $u$, runs the MG, chooses the nearest neighbor. Afterword, $u$ checks all the possible neighbors if they are inside the circle constructed. In addition, the algorithm behaves locally which means that the complexity is analyzed per one sensor node. For completeness, the MG's algorithm is included as shown in Algorithm 1 (appendix).

In the following section, we present the last framework's component, i.e., the routing sensors data.

\subsection{Routing of sensors data}

The proposed routing protocol has two phases: (1) setup and (2) steady phase. The setup phase includes zones creation, active nodes selection, and path establishment. All the framework's components are based on MG and GG. In the steady phase, data are transmitted from the source active node to the BS through intermediate active nodes. Active nodes at different levels work using TDMA scheme. The length of the timeslots at different levels is variable. For instance, at Timeslot 1, the active nodes at the farthest level $L_{k}$ sense the subscribed 


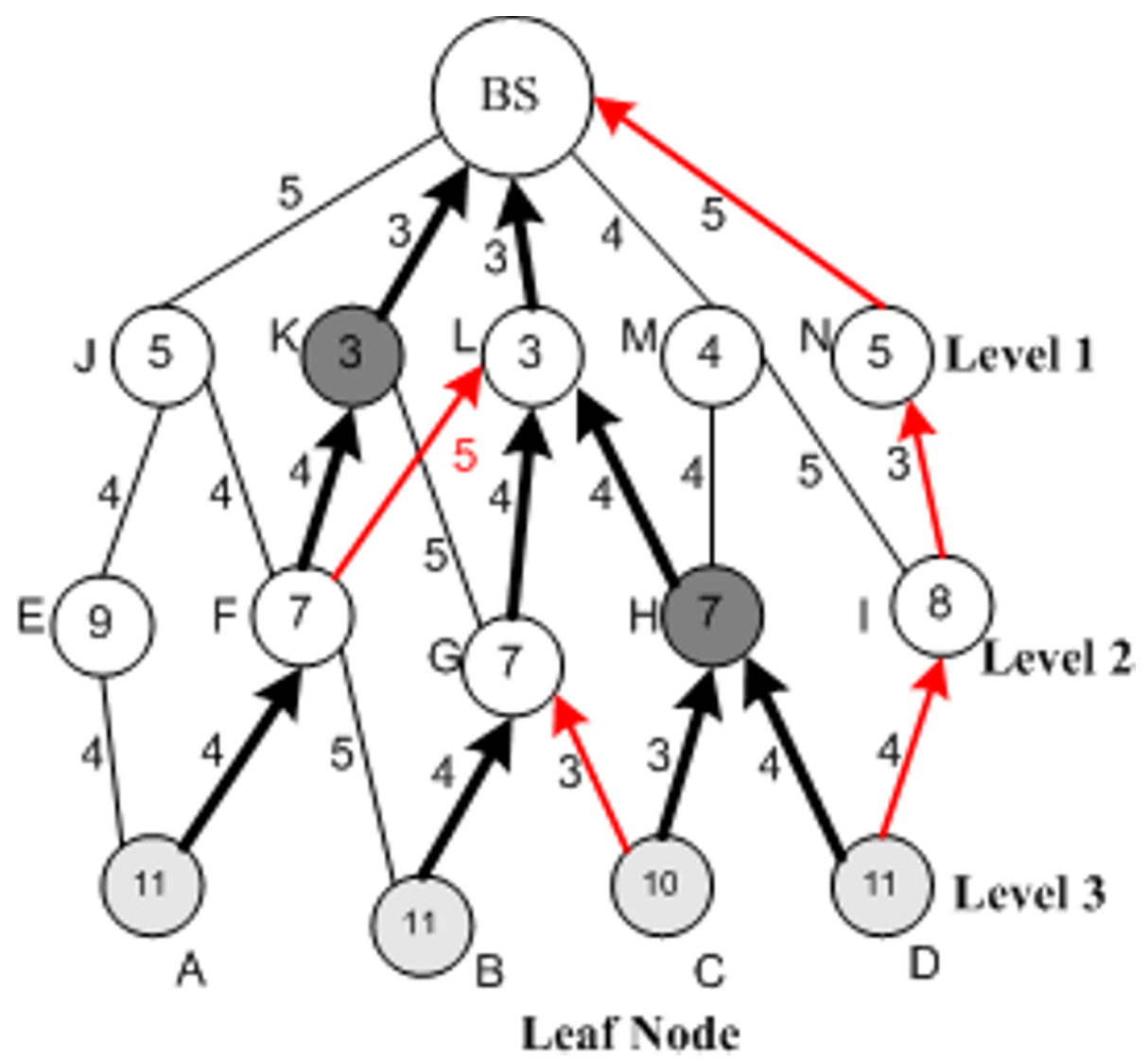

Figure 8 Alternative paths whenever a node fails.

events. Furthermore, the nodes send the corresponding data to active nodes at upper level $L_{k-1}$. At Timeslot 2, active nodes at the level $L_{k-1}$ receive data packet. These nodes send acknowledgements to nodes at the level $L_{k}$. Moreover, they transmit data packet to nodes at the upper level $L_{k-2}$. Hence, the length of Timeslot 1 is shorter than that of Timeslot 2.

The network is scalable since when more nodes are added to the network BS assigns zone ID to each node based on their locations and notifies $\mathrm{CH}$ without initiating any network setup phase. These nodes will be considered to be selected as active nodes whenever the setup phase is initiated after a certain number of rounds, numOfRound, based on the current network energy status.

$$
\text { numOfRound }=\frac{\text { prevNoOfRounds }}{\text { prevNetEnergy }} \times \text { currentNetEnergy }
$$

\section{Performance evaluation}

In this section, we present the energy model, the performance metrics, and the simulation model we have used for performance evaluation. Furthermore, we show the simulation results for the proposed CRTCA and its variant with Gabriel (GG) and MG graphs.

\subsection{Energy model}

The energy consumption of a sensor node for sending a data packet of size $n_{\text {data }}$ bytes to another sensor node (or $\mathrm{CH}$ ) at the distance $d$ is

$$
E_{\mathrm{TX}}=n_{\text {data }} \times \varepsilon_{\text {data }}+n_{\text {data }} \times d^{2} \times \varepsilon_{\text {air }}
$$

The energy consumptions of a node for receiving data

$$
E_{\mathrm{RX}}=n_{\text {data }} \varepsilon_{\text {data }}
$$

In Equation (2), $\varepsilon_{\text {data }}$ represents energy spent in transmitter electronics circuitry $(50 \mathrm{~nJ} / \mathrm{bit})$ and in Equation (3), $\varepsilon_{\text {air }}$ represents energy consumptions in radio amplifier for propagation loss $\left(10 \mathrm{pJ} / \mathrm{bit} / \mathrm{m}^{2}\right)$. Receiving energy consumptions of a sensor does not have energy consumptions of radio amplifier for propagation loss.

\subsection{Simulation model and performance metrics}

We simulate the performance of (1) CRTCA framework, (2) CRTCA-MG, and (3) CRTCA-GG using the C 
programming language. We use randomly connected UDGs on an area of $100 \mathrm{~m} \times 100 \mathrm{~m}$ as a basis of our simulation model. BS divides the network into a number zone and $\mathrm{CH}$ selects a number of active nodes in each zone that establish path to BS. The co-ordinate of the BS is assumed to be at $55 \times 101$.

The evaluation is done in terms of the following: (a) network energy consumptions (i.e., transmission and receiving power), (b) transmission energy consumptions, (c) end-to-end data transmission delay, (d) accumulated routing delays, and (e) length of the paths in terms of the crossed hops. Network energy consumption is defined as the total energy consumed by all the sensors nodes for routing data over a certain period of time. Network energy consumptions also reflect the lifetime of the network, i.e., the remaining network energy. We measure the end-to-end delay as the time that is required to transmit data from any source sensor node to the BS based on the traversed Euclidean distances. Another type of delay is in terms of the routing delay. Using this metric, the accumulated routing delays from any source sensor node to the BS are measured. Between these pairs of nodes, we also use another metric that counts the number of hops traversed on the path way which actually measures the network's paths lengths.

Some simulation runs measure these metrics by either varying the followings: (1) the number of rounds (num_r) (i.e., from 10000 to 40,000 of an increment of $10,000)$ and (2) the number of zones ${ }^{\mathrm{a}}\left(n u m \_z\right)$ (i.e., from 4 to 10 of an increment of 2). When varying num_r, we fix num_z and the number of sensor nodes (num_sens) to 4 and 120, respectively. However, when we vary the $n u m_{-} z$, we fix the num_r and the num_sens to 10,000 and 120 , respectively. For all these simulation runs, the constant $s$ for the MG graph is varied from 0.25 to 0.75 with an increment of 0.25 .
Lastly, the connectivity of the network based on the MG is determined by measuring the Largest Connected Component. If this component was equal to the total number of sensor nodes in the network, then the network would have to be connected.

\subsection{Simulation results}

Figure 9a,b illustrates the total network energy consumptions varying the number of zones in the network and number of rounds, respectively. In both cases, the CRTCA outperforms other approaches. The same observation applies even if $s$ is varied for the MG. This behavior is due to the high number of intermediate sensor nodes along the source-destination paths constructed by the CRTCA-GG and the CRTCA-MG. In other words, increasing the values of $s$ also increases the radius for the circular forbidden area where the intermediate nodes reside. Thus, more sensor nodes expose their battery power. As a consequence, the total network energy consumptions for the CRTCA-GG and the CRTCA-MG are more than that for the CRTCA. It is also evident that if there are more intermediate nodes on a path in GG and MG, the receptions energy consumptions at the intermediate nodes will contribute to the larger network energy consumptions.

Hence, we evaluate their performance in terms of only the transmission energy consumptions which are illustrated in Figure 10a,b. Again, we find that CRTCA outperforms the CRTCA-GG and CRTCA-MG. This is because more number of transmissions through the intermediate nodes will have more overhead energy consumptions of radio amplifier for propagation loss. Figure 11a,b supports the previous explanation by showing that the CRTCA maintains more number of shorter paths in terms of hops compared to those paths traversed by the other approaches.

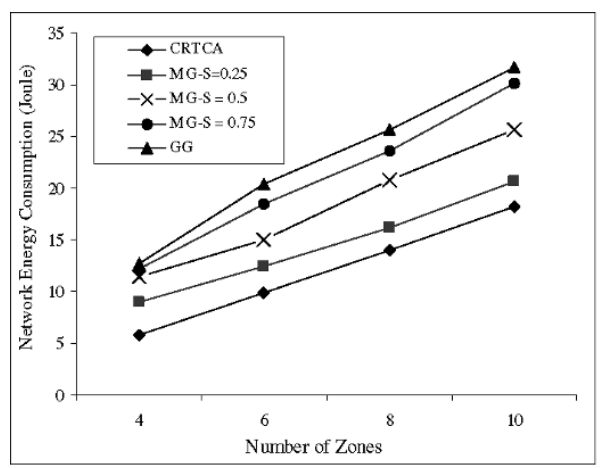

(a)

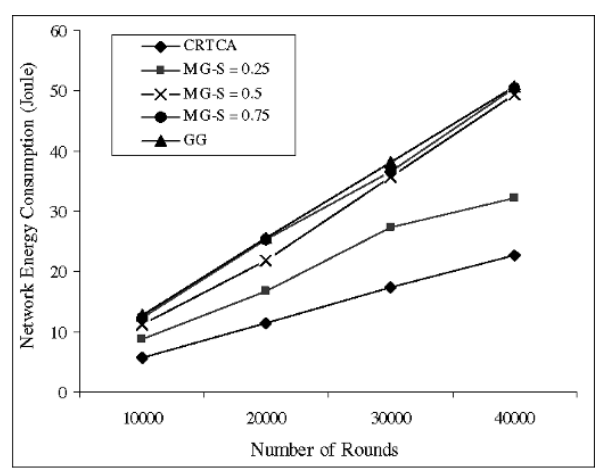

(b)

Figure 9 Network energy consumptions. 


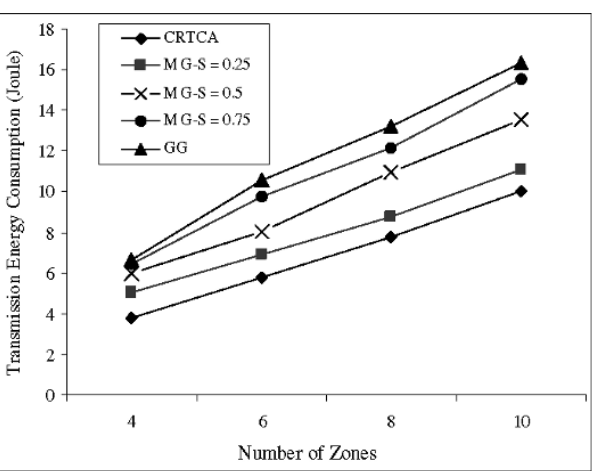

(a)

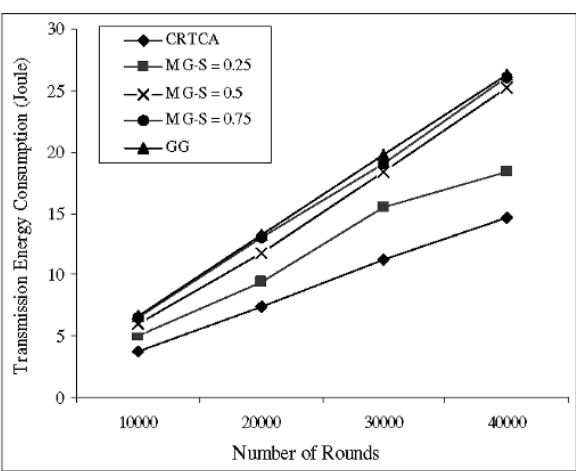

(b)

Figure 10 Transmission energy consumptions

From all the previous simulation runs, an interesting behavior for the set of MGs is demonstrated when $s$ is varied. The behavior is as follows: when $s$ increases the possibility for loosing edges increases as the radius for the circular forbidden area increases. As a consequence, more intermediate nodes are selected for transmitting data from a source node to the destinations that results more network energy consumptions, transmission energy consumptions, and number of hops.

In Figure 12a,b, we find that the CRTCA-MG and CRTCA outperforms the CRTCA-GG in terms of the end-to-end delay. This is because the MG keeps more edges compared to those for the GG. As a result, shorter paths are discovered by the CRTCA-MG which significantly improves the transmission delay.

Finally, Figure 13 demonstrates the routing delay for varying the number of zones. Again, CRTCA outperforms both MG and GG since in CRTCA, a path has less number of intermediate nodes and thus, delay for making routing decision will be less as compared to MG and GG which have more intermediate nodes on a data path.

In general, the above results demonstrate that CRTCA outperforms MG and GG in most cases. For instance, as the number of zones increases the number of levels in the hierarchy also increases. As a consequence, the number of sensor nodes is decreased in each subzone of a level. Therefore, the average degree of sensor nodes is decreased. Furthermore, because of the nature for the MG and the GG, more edges are removed. Thus, longer paths are achieved between any pair of sensor nodes in the GG and the MG that contribute to more network energy consumptions, transmission energy consumptions, and end-to-end delay. We also perform student's $t$-test at $95 \%$ confidence level which reveals the same phenomenon, as are demonstrated and stated. However, all these simulations demonstrate that the MG achieves the connectivity property.

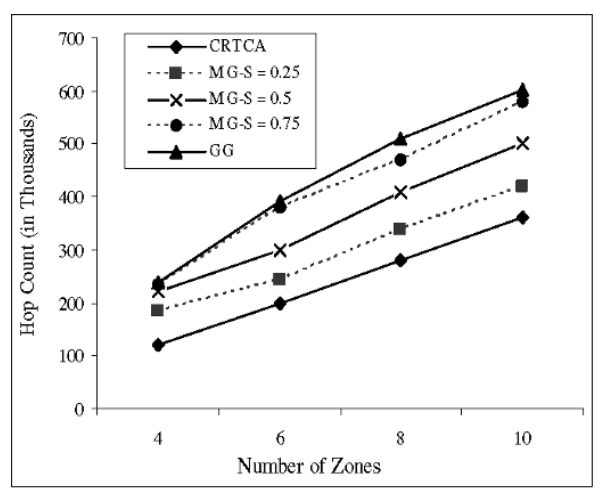

(a)

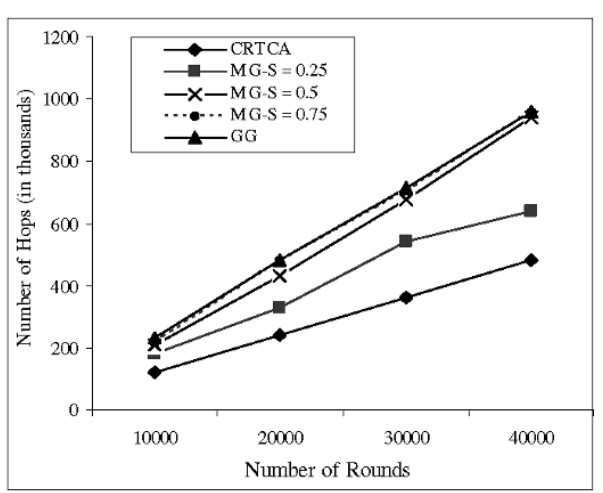

(b)

Figure 11 Paths lengths in terms of hops. 


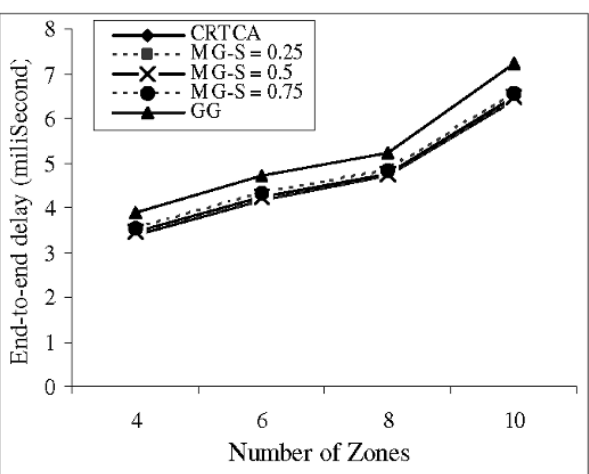

(a)

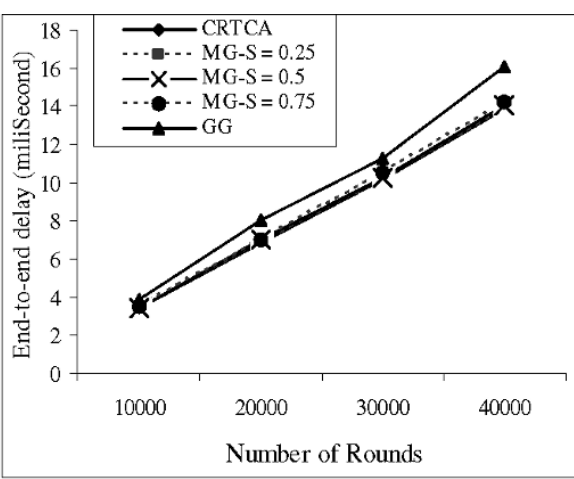

(b)

Figure 12 End-to-end data transmission delay

\section{Conclusion and future work}

In this article, we introduced a framework for WSN that combines clustering, routing, and topology control approaches. We refer to the framework as CRTCA. This framework uses an efficient zone-based topology and routing protocol. Moreover, a new set of graphs are used as underlying topologies for the framework referred to as the MG graph. We find that the CRTCA based on the proposed new set of MG graphs (CRTCAMG) outperforms the same framework based on an existing Gabriel graph (CRTCA-GG) in terms of the network energy consumptions, transmission energy consumptions, end-to-end data transmission delay, routing delay, and number of hops in the established path. In addition, the CRTCA demonstrates the best performance in most cases over CRTCA-MG and CRTCA-GG except the statistical analysis using student's $t$-test at 95\% confidence level reveals that both CRCTA and CRTCA-MG at lower value of $s$ have the same performance in terms of end-to-end delay. Moreover, the MG demonstrates that it achieves the connectivity property. In the future, we will theoretically prove some geometric properties for the MG graphs. Furthermore, we will evaluate the framework by varying the number of nodes

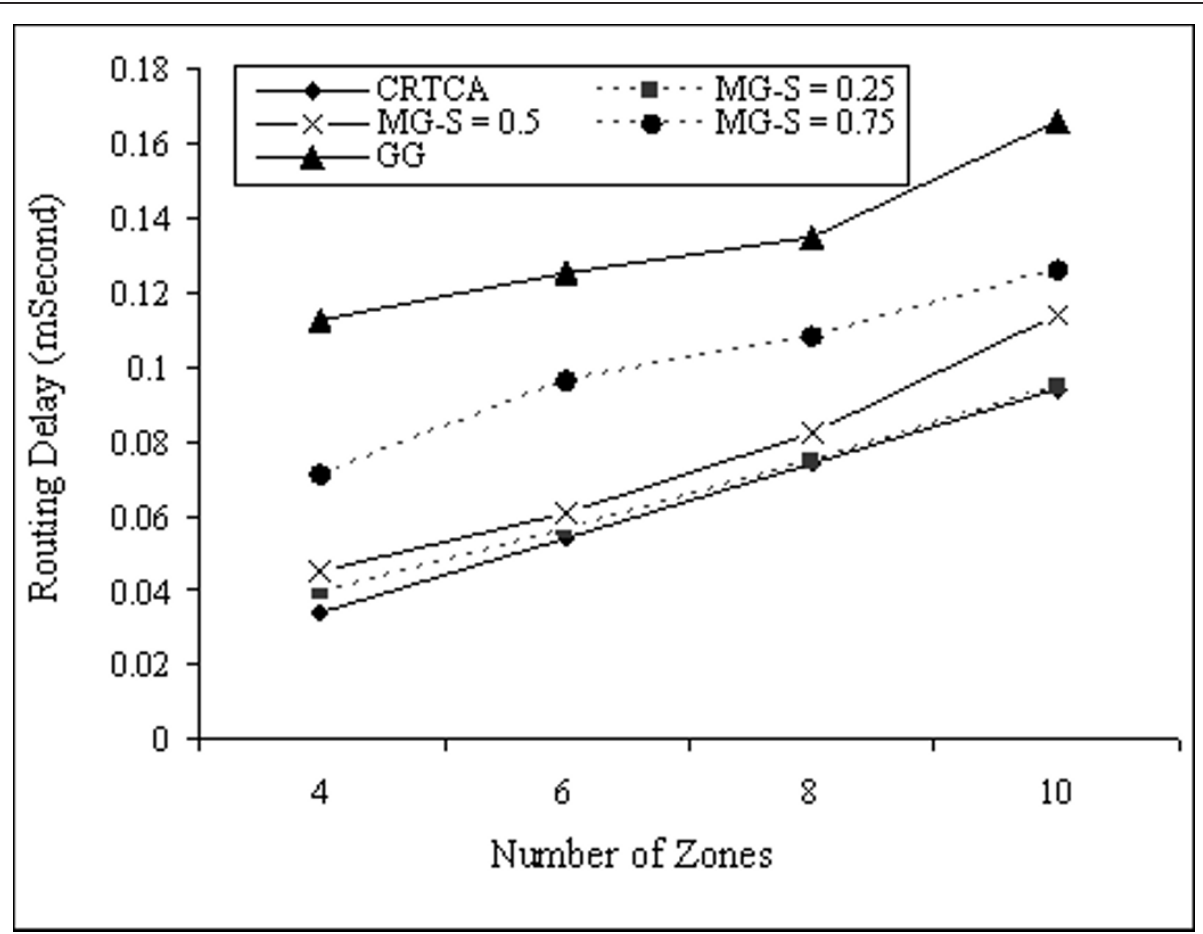

Figure 13 Routing delay varying number of zones. 
and the constant $s$ and in terms of additional defined metrics.

\section{Appendix}

Algorithm 1. MG(G, $s)$ graph algorithm

Input: A graph $G$ with the sensor node set $V$ and a parameter $s$.

Output: A list of undirected edges $L$ for each sensor node $u \in V$ which represents the MG subgraph of $G$, $\operatorname{MG}(G)$.

for all node $u \in V$ do

Create a list of neighbors of $u: L N(u)=N(u) . L=\varnothing$. repeat

(a) Remove the nearest neighbor sensor node $v$ from $L N(u)$.

(b) Scan the list $L N(u)$ and add the undirected edge $[u, v]$ to $L$ if $\nexists$ node $w$ that is inside the circle $c$ with a centre $=(u+v) / 2$ and a radius $=s .(|u v| / 2)$.

until $L N(u)$ is empty

endfor

\section{Endnote}

${ }^{\text {a It }}$ also implies the number of levels in the virtual hierarchical structure of WSN.

A short version of this paper (6 pages) has appeared in the proceedings of IEEE WiMob 2011.

\section{Author details}

${ }^{1}$ School of Computer Science, University of Guelph, Guelph, ON, Canada ${ }^{2}$ Electrical \& Computer Engineering Department, College of Engineering, Alfaisal University, Saudi Arabia

\section{Competing interests}

The authors declare that they have no competing interests.

Received: 30 October 2011 Accepted: 27 March 2012

Published: 27 March 2012

\section{References}

1. F Chiti, A De Cristofaro, R Fantacci, D Tarchi, G Collodo, G Giorgett, A Manes, Energy efficient routing algorithms for application to agro-food wireless sensor networks, in Proceedings of the IEEE International Conference on Communication (ICC), Seoul, Korea, pp. 3063-3067 (May 2005)

2. P Jacques, R Seshagiri, TV Prabhakar, H Jean-Pierre, HS Jamadagni, COMMONSense Net: a wireless sensor network for resource-poor agriculture in the semiarid areas of developing countries. J Inf Technol Int. 4(1), 51-67 (2007). doi:10.1162/itid.2007.4.1.51

3. S Yoo, J Kim, T Kim, S Ahn, J Sung, D Kim, A²S: automated agriculture system based on WSN, in Paper presented at the IEEE International Symposium on Consumer Electronics (ISCE), Dallas, TX, USA, pp. 1-5 (June 2007)

4. AH Kabashi, JMH Elmirghani, A technical framework for designing wireless sensor networks for agricultural monitoring in developing countries. in Paper presented at the International Conference on Next Generation Mobile Applications, Services and Technologies (NGMAST), Cardiff 395-401 (September 2008).

5. LK Kait, CZ Kai, R Khoshdelniat, SM Lim, EH Tat, Paddy growth monitoring with wireless sensor networks, in Paper presented at the International Conference on Intelligent and Advanced Systems (ICIAS), Kuala Lumpur, Malaysia, pp. 966-970 (November 2007)
6. Z Haas, M Pearlman, The performance of query control schemes for the zone routing protocol. IEEE/ACM Trans Netw (TON). 9(4), 427-438 (2001). doi:10.1109/90.944341

7. K Gabriel, R Sokal, A new statistical approach to geographic variation analysis. Syst Zool. 18, 259-278 (1969). doi:10.2307/2412323

8. E Chavez, S Dobrev, E Kranakis, J Opatrny, L Stacho, H Tejeda, J Urrutia, Half-space proximal: a new local test for extracting a bounded dilation spanner, in Proceedings of the International Conference On Principles of Distributed Systems, vol. 3974. Pisa, Italy, pp. 235-245 (2006)

9. G Toussaint, The relative neighborhood graph of finite planar set. Pattern Recogn. 12(4), 261-268 (1980). doi:10.1016/0031-3203(80)90066-7

10. D Matula, R Sokal, Properties of gabriel graphs relevant to geographic variation research and the clustering of points in the plane. Geograph Anal. 12(3), 205-222 (1980)

11. L Shu, Y Zhang, LT Yang, Y Wang, M Hauswirth, NX Xiong, TPGF: geographic routing in wireless multimedia sensor networks. Telecommun Syst. 44(1-2), 79-95 (2010). doi:10.1007/s11235-009-9227-0

12. S Liu, T Fevens, A Abdallah, Hybrid position-based routing algorithms for 3D mobile ad hoc networks, in Paper presented at the 4th International Conference on Mobile Ad-hoc and Sensor Networks, Wu Yi Mountain, China, pp. 177-186 (2008)

13. Z Yuan, L Wang, L Shu, T Hara, Z Qin, A balanced energy consumption sleep scheduling algorithm in wireless sensor networks, in Wireless Communications and Mobile Computing Conference (IWCMC), Istanbul, Turkey, pp. 831-835 (July 2011)

14. R Chen, Z Zhong, M Ni, Cluster based iterative GPS-free localization for wireless sensor networks, in Vehicular Technology Conference (VTC Spring), 2011 IEEE 73rd, Budapest, Hungary, pp. 1-5 (May 2011)

15. G Xing, C Lu, R Pless, Q Huang, Impact of sensing coverage on greedy geographic routing algorithms. IEEE Trans Parallel Distrib Syst. 17(4), 348-360 (2006)

16. W Yang, H Liusheng, W Junmin, X Hongli, Wireless sensor networks for intensive irrigated agriculture, in Paper presented at the Consumer Communications and Networking Conference (CCNC), Las Vegas, NV, USA, pp. 197-201 (January 2011)

17. W Ke, W Liqiang, C Shiyu, Q Song, An energy-saving algorithm of WSN based on gabriel graph, in 5th International Conference on Wireless Communications, Networking and Mobile Computing (WiCOM), Beijing, China, pp. 1-4 (September 2009)

doi:10.1186/1687-1499-2012-120

Cite this article as: Karim et al:: The significant impact of a set of topologies on wireless sensor networks. EURASIP Journal on Wireless Communications and Networking 2012 2012:120.

\section{Submit your manuscript to a SpringerOpen ${ }^{\mathcal{O}}$ journal and benefit from:}

- Convenient online submission

- Rigorous peer review

- Immediate publication on acceptance

- Open access: articles freely available online

- High visibility within the field

- Retaining the copyright to your article

Submit your next manuscript at $\boldsymbol{s p r i n g e r o p e n . c o m ~}$ 